\title{
Effect of vibration on muscle strength imbalance in lower extremity using multi- control whole body vibration platform ${ }^{1}$
}

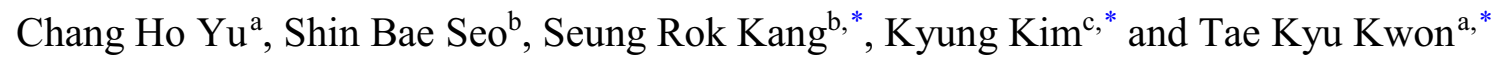 \\ ${ }^{a}$ Division of Biomedical Engineering, Chonbuk National University, 567 Baekje-daero, Deokjin-gu, \\ Jeonju-si, Jeonbuk 561-756, Republic of Korea \\ ${ }^{b}$ Department of Healthcare Engineering, Chonbuk National University, 567 Baekje-daero, Deokjin- \\ gu, Jeonju-si, Jeonbuk 561-756, Republic of Korea \\ ${ }^{c}$ Chonbuk National University Automobile-parts \& mold Technology Innovation Center, 67 Yusang-ro, \\ Deokjin-gu, Jeonju-si, Jeonbuk 561-844, Republic of Korea
}

\begin{abstract}
This study shows the improvement of muscle activity and muscle strength imbalance in the lower extremities through independent exercise loads in vibration platform. Twenty females of age 20 participated in this study. The subjects were divided into WBV group, with more than $10 \%$ of muscle strength imbalance between left and right the lower extremities, and control group, with less than $10 \%$ of muscle strength imbalance between left and right the lower extremities. As the prior experiment showed, different exercise postures provide different muscular activities. As a result, the highest muscular activity was found to be in the low squat posture. Therefore, the LS posture was selected for the exercise in this experiment. Vibration intensities were applied to dominant muscle and non-dominant muscle, and the vibration frequency was fixed at $25 \mathrm{~Hz}$ for the WBV group. The control group was asked to perform the same exercise as the WBV group, without stimulated vibration. This exercise was conducted for a total of 4 weeks. As a result, the WBV group which showed an average deviation of $16 \%$ before the experiment, tended to decrease approximately to $5 \%$. In this study, vibration exercise using load deviation is shown to be effective in improving the muscle strength imbalance.
\end{abstract}

Keywords: Whole body vibration, load deviation, muscle strength imbalance, surface EMG

\footnotetext{
${ }^{1}$ None of authors received financial support or have relationships that may pose a conflict of interest.

*Address for correspondence: Tae Kyu Kwon, Division of Biomedical Engineering, Chonbuk National University, 567 Baekje-daero, Deokjin-gu, Jeonju-si, Jeonbuk 561-756, Republic of Korea. Tel.: 063-270-4066; Fax: 063-270-2247; E-mail: kwon10@jbnu.ac.kr.

Seung Rok Kang, Department of Healthcare Engineering, Chonbuk National University, 567 Baekje-daero, Deokjin-gu, Jeonju-si, Jeonbuk 561-756, Republic of Korea. Tel.: 063-270-4066; Fax: 063-270-2247; E-mail: okokman@naver.com.

Kyung Kim, Chonbuk National University Automobile-parts \& mold Technology Innovation Center, 67 Yusang-ro, Deokjin-gu, Jeonju-si, Jeonbuk 561-844, Republic of Korea. Tel.: 063-219-0322; Fax: 063-219-0311; E-mail: kkim@camtic.or.kr.
} 


\section{Introduction}

Resistance exercise has been known as the main technique for the improvement of muscle strength and power [1]. Over the past few years, various researches on resistance exercise and methods have made constant progress [2]. In particular, many studies have reported that the muscle strength imbalance is a cause of backache or vertebral disease and also a cause of falling in the aged people. The muscle strength imbalance may reduce one's ability to perform the exercise or physical activities, and it is usually caused by incorrect exercise [3]. Also, it can be the cause of an imbalanced posture, ultimately reducing the flexibility of the body. The physical abnormalities, obesity, or chronic degeneration can be caused by strained conditions during the physical development [4]. Many ongoing studies are currently focused on resolving the increasing injuries or diseases caused by physical imbalance [5]. However, the studies on muscle strength imbalance are at the beginning stage, and the treatment or improvement is still unknown. Among the different solutions suggested by various ongoing studies, the whole body vibration emerged with positive outlook, which gives fewer burdens to the body and more impacts on muscle strength [6].

The whole body vibration (WBV) can develop the entire muscular system, rather than concentrating on a specific area. It was newly introduced in 1990s as a new method for training the muscular function that is widely being used in various settings including sports team, rehabilitation clinics, and fitness clubs, especially in the advanced countries of modern days [7, 8]. In a study on whole body vibration conducted by Bosco [9], it has been reported that WBV showed a positive impact on neuromuscular improvement in 14 males of age 20. Baik [10] also proved the positive effect of temporary WBV as a warm-up exercise and its improvement in agility. Delecluse [11] suggested that the whole body vibration increased muscle strength in the knee joint extension, compared to general muscle exercise, and this result was shown with 67 adult females who performed the whole body vibration exercise for 12 weeks. Freddy [12] reported that the whole body vibration is an effective exercise to improve the posture balance and walking ability and it prevents accidental falling of the aged people. Roelants [13] reported that the whole body vibration did not change the physical composition in the group of young females with no previous experiences on physical exercise, when exercised for 24 weeks. However, this vibration exercise induced the increase of muscle strength in the extension of the knee joint. Kawanabe [14] reported that the whole body vibration is an affective exercise to improve the muscular strength, balance ability, and waking ability for the aged group. However, a study in the effect of whole body vibration on the adjustment of muscle strength imbalance is surprisingly insignificant. Also, a study on the system development for adjusting the muscle strength imbalance is nonexistent.

Therefore, this study was conducted to investigate the effect of exercise load deviation on the adjustment of muscle strength imbalance in lower extremities, by independently providing the existing vertical whole body vibration to both left and right lower extremities.

\section{Experimental methods}

\subsection{Subjects}

Ten (10) females in their 20s, without a medical history of musculoskeletal injuries in the last 6 months, with prior experiences in WBV, and with deviation of muscular strength imbalance between left and right lower extremities of more than 10\% [15], were assigned into WBV group. Another group 
Table 1

Subject information in the WBV and Control Group

\begin{tabular}{lll}
\hline & WBV Group & Control Group \\
\cline { 2 - 3 } Age & $21 \pm 3.6$ year & $21 \pm 0.8$ year \\
\hline Height & $159 \pm 5 \mathrm{~cm}$ & $157 \pm 3.2 \mathrm{~cm}$ \\
\hline Weight & $47 \pm 4.6 \mathrm{~kg}$ & $47 \pm 5.8 \mathrm{~kg}$ \\
\hline Deviation & $16.8 \pm 3.4 \%$ & $3.6 \pm 1.6 \%$ \\
\hline
\end{tabular}

of ten (10) females with deviation of muscular strength imbalance between left and right lower extremities of less than $10 \%$ were assigned into control group (Table 1). Conforming to the Declaration of Helsinki (1964), written informed consent was obtained from all subjects. Also, the exercise postures causing the muscular strength imbalance of the lower extremities in control group were analyzed.

After confirming that all subjects have no problem with whole body vibration, we also considered that the subjects had similar body weight, height, eating habits, body type, and exercise habits, to reduce the error of the experimental environment. All vibration and control protocols were performed on a commercial vibrating platform that has been factory validated (Sonics-VM10, Sonicworld, Co., Ltd., Korea). This platform produces up-to-down alternating vertical sinusoidal vibration.

\subsection{System configuration}

A multi-control whole body vibration platform (manufactured by Sonicworld. Ltd., Korea) was used to conduct this study, as shown in Figure 1. It enables independent control of four vibration platforms and provides a diverse range of vibration frequency from 0 to $50 \mathrm{Hzm}$ and amplitude (intensity) from 0 to $9 \mathrm{~mm}$. Also, this platform was manufactured by applying an intuitive interface that uses a touchscreen monitor.

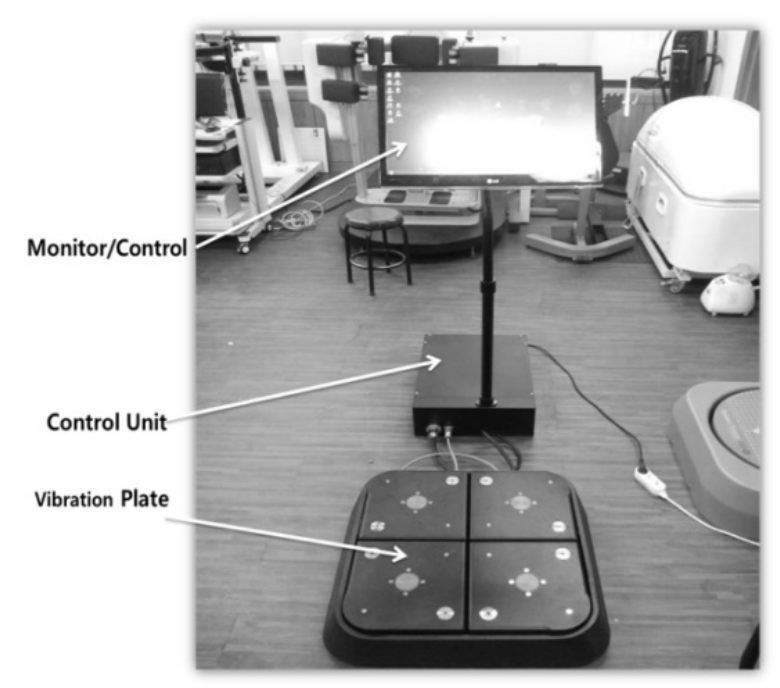

Fig. 1. Multi-control whole body vibration platform (Sonicworld. Ltd., Wonju, Korea). 


\subsection{Experimental equipment and procedures}

The subjects were divided into two groups; WBV group was provided with a new whole body vibration and the load deviation during their exercise, and the control group was not provided with stimulated vibration during their exercise. The exercise posture causing the greatest muscle strength imbalance of the lower extremities was revealed in the prior experiment. All subjects were asked to perform the exercise for 4 weeks, 3 times a week, and 15 times of 5 sets a day. BIODEX System 3 (Biodex Medical System Inc., NY, USA) was used to measure and analyze the knee joint torque prior to the experiment, and 2 and 4 weeks after the experiment $[16,17]$. Then, the deviation of muscular strength between left and right lower extremity was analyzed.

The subject's posture during the squat exercise was adjusted, in order to control the factors affecting the effect of exercise. To reduce the errors from environmental factors, room temperature and humidity were constantly maintained at $20^{\circ} \mathrm{C}$ and $45-55 \%$, respectively, while subjects were exercising and being evaluated. The subjects were asked to perform the same motion of exercise to reduce the errors in experimental outcomes caused by differences in each subject's posture. The presence of adverse reaction in the subjects was confirmed prior to their every exercise to confirm the absence of the subject's side effects from the equipment.

\subsection{Experimental methods}

As shown in Figure 2, the control group was asked to take off their shoes to stand on the vibration platform, and perform four different squat motions taken from the prior experiment. The four squat motions include low squat (LS: knee joint flexion at $90^{\circ}$ ), high squat (HS: Knee joint flexion at $125^{\circ}$ ), and wide squat (WS: knee joint flexion at $90^{\circ}$ ), which were done for 20 seconds each [18].

For variation of vibration, both stimulated and non-stimulated vibrations were suggested to be done for 10 seconds. The suggested frequency was set at $25 \mathrm{~Hz}$ in this study, which is the average of the frequencies used in the existing studies [19-26]. A diverse range of vibration intensities from 0 to 9 $\mathrm{mm}$ was available by this equipment, and $4 \mathrm{~mm}$ was found to induce the highest muscular activity.

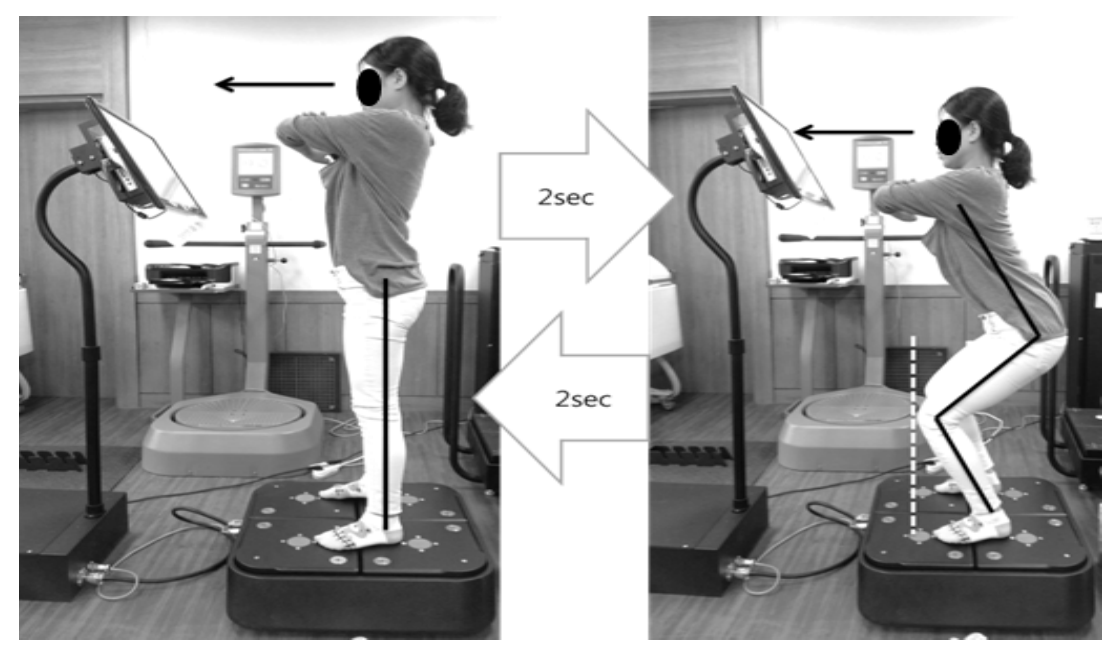

Fig. 2. Squat exercise using load deviation type of the whole body vibration platform. 

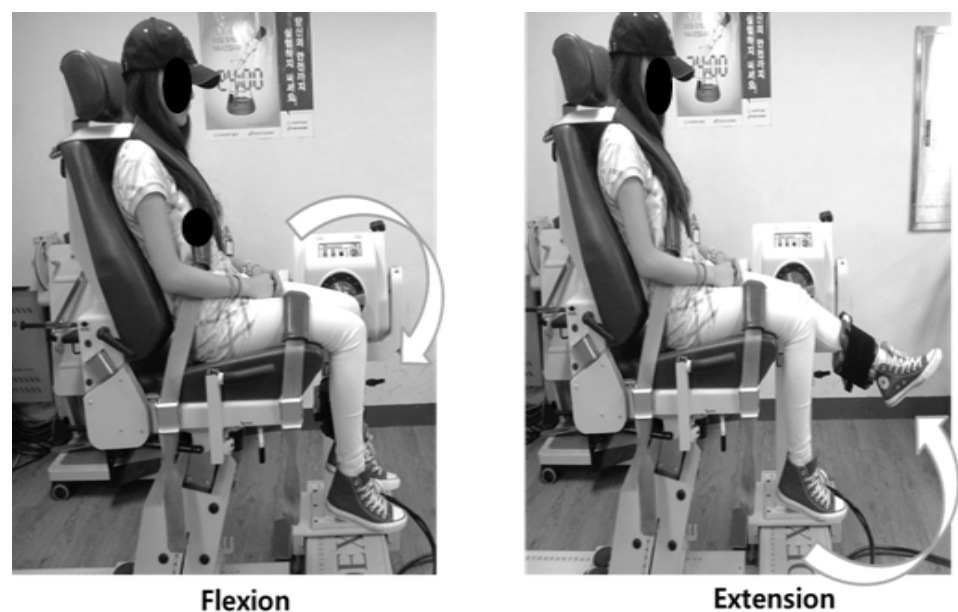

Fig. 3. Evaluation of joint torque in knees for observing the difference of muscle strength using BIODEX System 3.

As shown in Figure 3, the BIODEX System3 (Biodex medical science, NY, USA) was used as a tool to evaluate the differences in the function of isokinetic muscular strength in lower extremities. By these means, the changes of knee joint torque were observed before the experiment and two and four weeks after the experiment. The knee joint was measured to observe the changes in joint torque occurring at the dorsiflexion and flexion, before and after the exercise. The angular velocity measured to evaluate the changes in muscular strength was $60^{\circ} / \mathrm{sec}$, and the range of motion (ROM) was $60^{\circ}$, which were equally applied to all subjects.

For surface EMG assessment, Bagnoli-EMG 8ch (Delsys Inc., NY, USA) was used to measure the rectus femoris (RF), vastus lateralis (VL), and vastus medialis (VM). Based on the results of the prior experiment, the low squat (LS) motion causing higher activity and imbalance was performed in this experiment. The subjects were instructed to straighten their backs, look straight ahead, and not to widen the width between their knees. They were instructed to bend and stretch their knees for two seconds. Also, a metronome was used to control the exact exercise time, so the same time and intensity were provided to all subjects. The vibration frequency in WBV group was fixed at $25 \mathrm{~Hz}$. The load deviation was provided by fixing the intensity of vibration for dominant muscle strength at $1 \mathrm{~mm}$ and the non-dominant muscle strength at $4 \mathrm{~mm}$.

\subsection{Data analysis}

The EMG signals were collected in a real-time manner at $1000 \mathrm{~Hz}$ to analyze the changes of characteristics in muscular activity, following a diverse number of squat postures. Also, a bandwidth filter $(20-450 \mathrm{~Hz})$ was used to remove noise.

In order to confirm the statistical significance, an SPSS 18.0 kor (SPSS Inc, Chicago, USA) was used to calculate the average and standard deviation for real-time EMG RMS data, while the subject was doing a squat motion. The average and standard deviations of the knee joint torque were calculated to prove the improvement on muscular imbalance. A test of normality was performed on all results through the Kolmogorov-Smimov test. Two sample $\mathrm{T}$ tests were performed to prove the changes in muscular ability from stimulated and non-stimulated vibration and the significance of improvement in muscular imbalance. The significance level was $* \mathrm{p}<0.05$. 


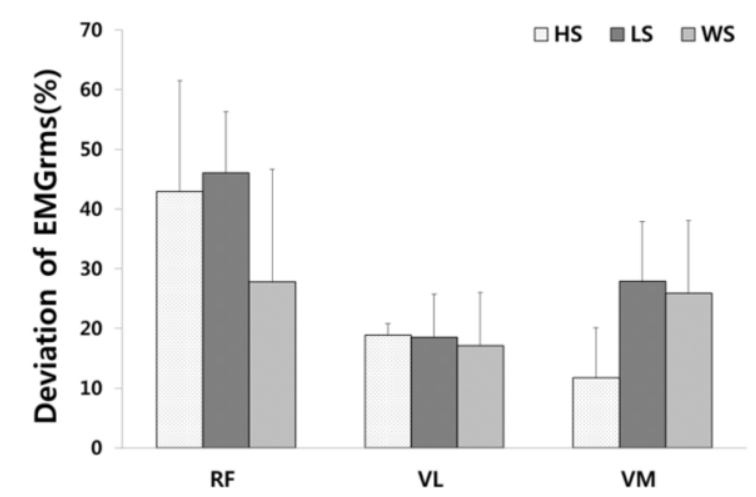

(a)

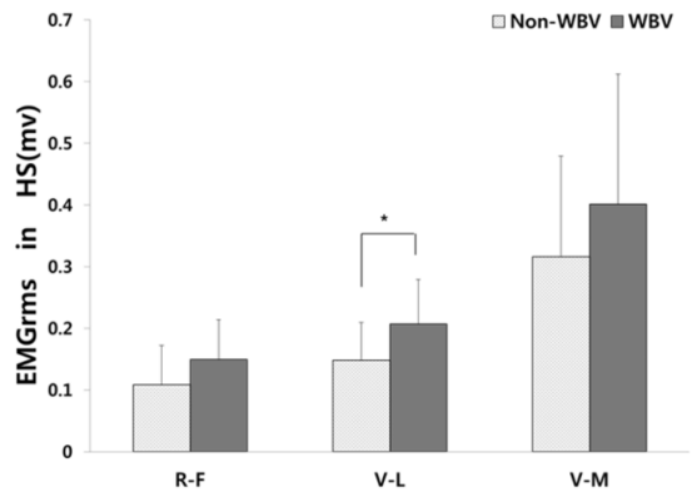

(b)

Fig. 4. (a) Deviation of electromyography in the leg muscles adapting to load deviation techniques, before the exercise (b) Changes in muscle activity on the high squat (mean $\pm \mathrm{SD},{ }^{*} \mathrm{p}<0.05$ ).

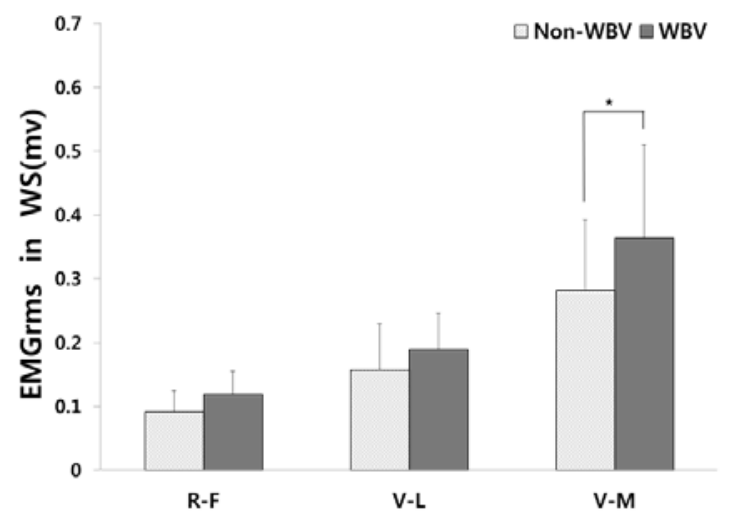

(a)

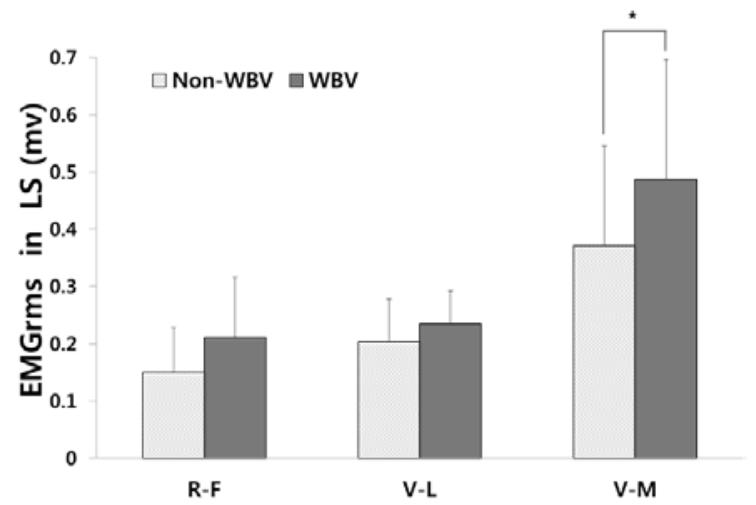

(b)

Fig. 5. (a) Changes in muscle activity on the wide squat (b) Changes in muscle activity on the low squat (mean $\pm \mathrm{SD}$, $* \mathrm{p}<0.05)$.

\section{Results}

The results of evaluation on muscular ability in both lower extremities (left and right) at three different squat motions are shown in Figure 4(a). The deviations of muscular activity while performing a high squat were shown to be as follows: $42.9 \%$ in the rectus femoris, $18.8 \%$ in the vastus lateralis, and $11.7 \%$ in the vastus medialis. The deviations of muscular activity while performing a low squat were shown to be as follows: $46 \%$ in the rectus femoris, $18.5 \%$ in the vastus lateralis, and $27.8 \%$ in the vastus medialis. The deviations of muscular activity while performing a wide squat were shown to be as follows: $27.7 \%$ in the rectus femoris, $17.1 \%$ in the vastus lateralis, and $25.9 \%$ in the vastus medialis.

Figures 4(b), 5(a) and 5(b) show the changes of muscular activity in leg muscles (rectus femoris, vastus lateralis and vastus medialis) at three different squat motions, with and without WBV. The 
increase of muscular acitivity on the rectus femoris muscle while providing WBV was shown to be as follows: $27 \%$ in HS motion, $23 \%$ in WS motion, and $28 \%$ in LS motion. The increase of muscular acitivity on the vastus lateralis muscle while providing WBV was shown to be as follows: $28 \%$ in HS motion, $17 \%$ in WS motion, and $13 \%$ in LS motion. The increase of muscular activity on the vastus medialis muscle while providing WBV was shown to be as follows: $22 \%$ in HS motion, $22 \%$ in WS motion and $23 \%$ in LS motion.

The highest increase of muscular activity in all measured muscles was found in LS motion amongst all three different motions. Also, the imbalance of subjects' muscular activity was found to be the greatest in LS posture. The LS motion induces the greatest amount of muscular activity, compared to the other motions, due to its higher level of difficulty. Based on these results, LS posture was selected in this study.

Table 2 shows the results of the effects of vibration exercise with applied load deviation on the improvement of muscular strength imbalance in the lower extremities. Figure 6(a) shows the result of analyzing peak torque. The WBV group had a deviation of about $16 \%$ in the lower extremities muscular strength before the experiment, which significantly decreased to around $5.2 \%$. Whereas, the control group, without stimulated vibration, had a deviation of about $4.0 \%$ before the experiment, which increased to $5.3 \%$ after four weeks. A significant decrease of $69.1 \%$ was shown in the WBV group, indicating the positive effect of improving muscular strength imbalance. A peak torque refers to measuring the strength of the muscle.

Figure 6(b) shows the result from analyzing the average power in the WBV group, in which the deviation of $23 \%$ before the experiment decreased to $5.6 \%$ after four weeks. Whereas in the control group, the initial deviation of about $3.0 \%$ measured before the experiment increased to $6.9 \%$ after four weeks. The WBV group using the exercise load deviation had significantly decreased by $75 \%$, showing a positive result that the muscular strength imbalance had improved. The average power is the value of the total completed work divided by the total days. This implies how fast the muscle strength can be produced. This is used as an indicator for the evaluation of muscular reactivity.

Table 2

Deviation of knee joint muscle strength adapting load deviation methods, before and after exercise (mean $\left.\pm \mathrm{SD},{ }^{*} \mathrm{p}<0.05\right)$

\begin{tabular}{lllllllll}
\hline & \multicolumn{7}{l}{ WBV Group (\%) } & \multicolumn{7}{c}{ CON Group (\%) } \\
\cline { 2 - 10 } & Pre & Post & Mean & p-value & Pre & Post & Mean & p-value \\
\hline Peak Torque & 16.8 & 5.2 & 10.9 & $0.003^{*}$ & 4.0 & 5.3 & 3.8 & 0.332 \\
\hline AVG Power & 23.4 & 5.6 & 18.5 & $0.004^{*}$ & 3.0 & 6.9 & 3.2 & 0.370 \\
\hline Total Work & 17.8 & 7.1 & 17.3 & $0.004^{*}$ & 4.4 & 6.9 & 4.2 & 0.198 \\
\hline Acceleration Time & 27.1 & 12.3 & 12.1 & $0.028^{*}$ & 15.7 & 7.8 & 8.4 & 0.437 \\
\hline
\end{tabular}

Note: WBV group: whole body vibration exercise group, CON group: non-vibration group

Pre: Test results before the experiment, Post: the results when joint torque was evaluated after the experiment

$\mathrm{P}$-value is given for the overall difference between the groups, according to ANOVA

Significant intra-group-differences as calculated using paired t-tests are marked with asterisks: mean $\pm \mathrm{SD},{ }^{*} \mathrm{P}<0.05$.

Figure 6(c) shows the result of analyzing the amount of total work. The WBV group had a deviation of $17.8 \%$ before the experiment, which decreased by around $59 \%$ to around $7.1 \%$ after the experiment. This is the value multiplied by the distance of the joint working range that was equally set for torque, 
by the exercise direction and strength direction, during the extension of knee joint. In other words, this suggests that the muscles in both left and right lower extremities were being used equally.

Figure 6(d) shows the result of analyzing the muscular velocity. The velocity is the time taken from the starting point of knee joint torque to the threshold of velocity, with the sum of angles while performing knee joint extension and flexion exercise, which was done five times in a row; thereby, this velocity refers to the ability of nervous muscle to move from a steady state to isokinetic velocity. As a result, the WBV group with a deviation of around $27 \%$ before the experiment had decreased by $54 \%$ to around $12.3 \%$, after the experiment. These results suggest that the stimulation of the whole body vibration continuously stimulates the muscular nerve at the contraction of the muscle that are responsible for the extension and flexion of the knee joint, and induces muscle tension which improves the muscular acceleration time.

\section{Discussion}

This study seeks to prove the effect of whole body vibration on the adjustment of muscle strength imbalance. As a result of the prior experiment, the highest muscular activity as well as the greatest

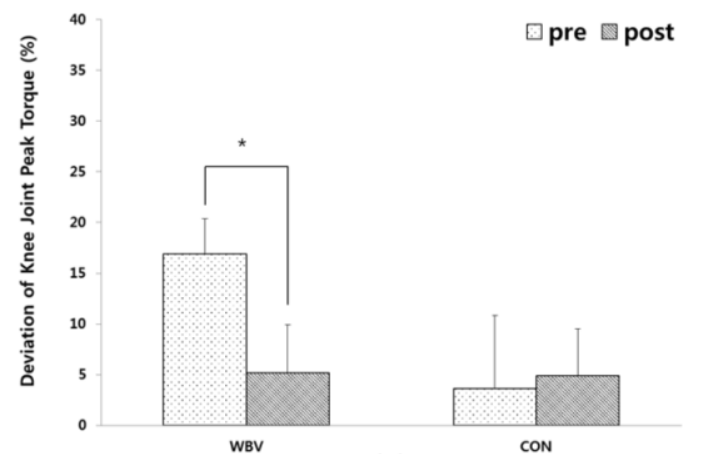

(a)

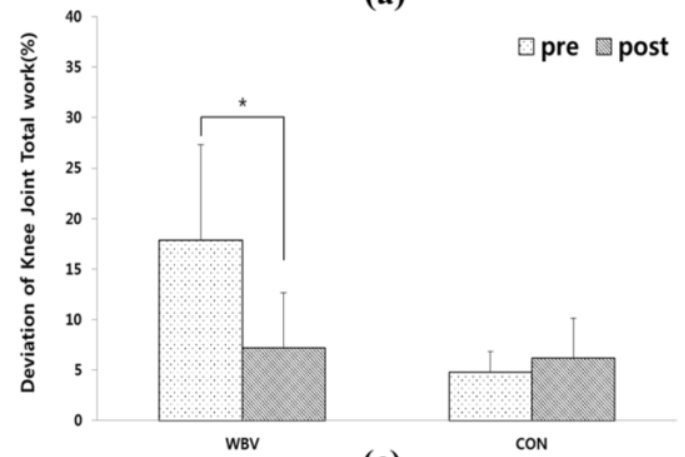

(c)

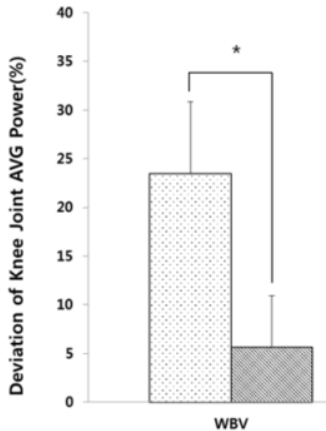

(b)

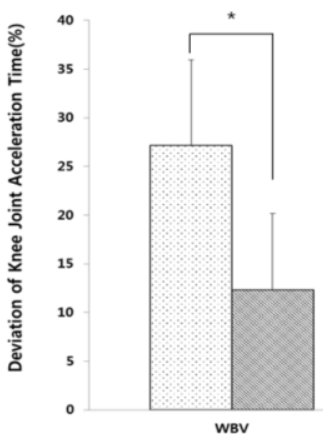

(d) pre $\square$ post

pre post

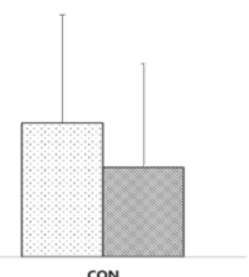

Fig. 6. Variation of difference in peak torque(a), average power(b), total work(c) and acceleration time(d) in knee joint before and after 4 weeks (mean $\pm \mathrm{SD},{ }^{*} \mathrm{p}<0.05$ ). 
imbalance of muscular strength was found in LS motion. This suggests that LS motion induces more muscular activities compared to other motions, due to its higher level of difficulty. Furthermore, it causes the imbalance of muscular activity, by exerting greater strength on the lower extremity than commonly used by habit.

The vibration exercise using load deviation proved its effect on the improvement of muscular strength imbalance in the lower extremities in the WBV group, from performing the exercise at different intensity on the left and right vibration platform.

The WBV group, whose average deviation was $16 \%$ before the experiment, showed a deviation of $4.7 \%$ after the experiment. The control group, whose deviation was $3.2 \%$ before the experiment, showed a deviation of $5.9 \%$ after the experiment. About $70 \%$ of imbalance was reduced in the WBV group. This suggested that the muscular strength imbalance can be adjusted, if exercise load deviation was independently applied to the left and the right lower extremities. Also, the exercise load provided in this tool was considered to enable the dominant and non-dominant muscle in the lower extremes to indecently exercise. As a result, the muscle strength imbalance has been fixed to balance, as described above. In other words, this suggests that the dominant muscle in the lower extremities performs the exercise that enhances muscular endurance, whereas the non-dominant muscle in the lower extremities performs the exercise that enhances strength $[3,27]$.

For the average power, the WBV group with a deviation of about $23 \%$ before the experiment, tended to decrease to around $5.6 \%$ after the experiment. In the control group, the deviation before the experiment was about $2.4 \%$ which resulted to be about $5.1 \%$, after the experiment. The WBV group that used the exercise load deviation tended to significantly decrease by $75.9 \%$. Also, for the muscular acceleration time, the deviation of $27 \%$ in WBV group before the experiment had decreased by $54 \%$ down to $12.3 \%$ after the experiment.

These results suggest that the stimulation of the whole body vibration continuously stimulates the muscular nerve at the contraction of muscles responsible for extension and flexion of the knee joint, and causes muscular tension which improves the muscular acceleration time. In other words, the stimulated vibration stimulates the muscle spindles and induces the reflective contraction of the muscles. Therefore, it increases the muscular activity and reactivity when stimulated vibration is applied [28].

The muscular strength imbalance between the left and the right extremities is still undefined, and the relevant studies are still inadequate. Most of the studies are focused on the enhancement of muscular strength, muscular damage or fatigue. The enhancement of muscular strength is more focused on making them stronger through typical exercise, rather than balancing the muscular strength. On the other hand, this study focused on the balancing development of muscular strength. The rowing machine also uses load deviation and shows similar trend on the improvement of muscular strength imbalance in the upper extremities [5]. In this study, the subjects who initially showed more than $15 \%$ of muscular strength imbalance in the lower extremities decreased to less than $10 \%$ after four weeks. This suggests that the exercise providing an independent load induces the development of the muscular nervous system, giving positive effects to the balanced development of muscular strength and muscular reactivity. However, the control group that showed the normal deviation of muscular strength between left and right lower extremities of within 5\% had increased deviation after four weeks. This suggests that the general squat exercise uses more of the dominant muscle by habit, causing the imbalance of muscular strength.

The whole body vibration exercise would be greatly helpful in improving an athlete's performance, through developing an effective exercise protocol that evenly develops the entire muscles in a body and not only the lower extremities. Also, this whole body vibration exercise is considered to be 
effective without straining the body. Therefore, this can be used as part of the rehabilitation exercise for patients having muscular strength imbalance due to a long-term injury. Furthermore, it has been reported that the whole body vibration induces improvement of the neuromuscular system and enhances muscular strength in the lower extremities without straining the body, both in the aged and healthy adults $[29,30]$.

\section{Conclusion}

This study was done to prove the effect of multi-control whole body vibration on the adjustment of muscular strength imbalance in the lower extremities. As a result, the following conclusion has been drawn.

Vibration exercise using the load deviation improves the muscle strength imbalance in the lower extremities, and it improves the muscular reactivity.

This suggests that the stimulated whole body vibration stimulates the muscle spindles to induce higher muscular activity, giving a positive effect on muscular development, to improve the muscle strength imbalance and reactivity in the lower extremities

As a result of this study, this multi-control whole body vibration can be applied as part of a rehabilitation program or exercise prescription for healthy, aged, or athletic people with muscle strength imbalance due to injuries in the musculoskeletal system.

\section{Acknowledgments}

This research was financially supported by the Ministry of Trade, Industry and Energy (MOTIE) and Korea Institute for Advancement of Technology (KIAT) through the Promoting Regional Specialized Industry (No. R0002430) and partially supported by Basic Science Research Program through the National Research Foundation of Korea (NRF) funded by the Ministry of Science, ICT and Future Planning (No. 2014R1A1A1006266).

\section{References}

[1] J.W. Park, K.N. Kim and D.H. Hong, Haptic based resistance training machine and its application to biceps exercises, International Journal of Precision Engineering and Manufacturing 12 (2011), 21-30.

[2] J.W. Park, K.N. Kim, D.H. Hong, J.H. Moon, D.H. Koo, M.S. Kang, I.S. Shin, Y.H. Kim and K.W. Lee, The shoulder abduction exercise with a haptic-based resistance training machine, International Journal of Precision Engineering and Manufacturing 13 (2012), 2239-2243.

[3] R. Chou, S.J. Atlas, S.P. Sanons and R.W. Rosenquist, Nonsurgical interventional therapies for low back pain: A review of the evidence for an American pain society clinical practice guideline, Spine 34 (2009), 1078-1093.

[4] K.W. Lee, J.H. Hwang and H.J. Bang, Isometric evaluation of the lumbar extensors in chronic low back pain, Korean Academy of Rehabilitation Medicine 21 (1997), 1-7.

[5] S.R. Kang, S.B. Seo, G.Y. Jeong, J.J. Bae, C.H. Yu, M. Yu, D.A. Moon, J.S. Jeong and T.K. Kwon, Effect on improvement of muscle strength imbalance according to load deviation pattern of left and right arms in upper limbs, Journal of Korean Society Precision Engineering 29 (2012), 1026-1034.

[6] S.R. Kang, D.A. Moon, C.H. Yu and T. K. Kwon, Effect of long whole-body vibration training on muscle function and postural Balance, International Journal of Precision Engineering and Manufacturing 15 (2014), 1681-1688.

[7] Y.T. Lim, Study on whole body vibration as a new exercise training prescription method, Journal of Coaching Development 7 (2005), 105-116. 
[8] M. Cardinale and T. K. Kwon, Fundamental study of lower limb muscle activity using an angled whole body vibration exercise instrument, Bio-Medical Engineering Materials and Engineering 24 (2014), 2437-2445.

[9] C. Bosco, The use of vibration as an exercise intervention, Exercise and Sport Science Reviews 31 (2000), 3-7.

[10] C. Bosco, M. Iacovelli, M.O. Tsarpela, M. Cardinale, M. Bonifazi, J. Tihani, M. Viru, A. de Lorenzo and A. Viru, Hormonal responses to whole body vibration in men, European Journal of Applied Physiology 81 (2000), 449-454.

[11] S.W. Baik, The effect of whole body vibration exercise for warm up, Journal of Sport and Leisure Studies 49 (2012), $729-736$.

[12] C. Delecluse, M. Roelants and S. Verschueren, Strength increase after whole body vibration compared with resistance training, Medicine \& Science in Sports \& Exercise 35 (2003), 1033-1041.

[13] F.M. Lam, R.W. Lau, R.C. Chung and M.Y. Pang, The effect of whole body vibration on balance, mobility, and falls in older adults: A systematic review and meta-analysis, Maturitas 72 (2012), 206-213.

[14] M. Roelants, C. Delecluse, M. Goris and S. Verschueren, Effects of 24 weeks whole body vibration training on body composition and muscle strength in untrained females, International Journal of Sports Medicine 25 (2004), 1-5.

[15] K. Kawanabe, K. Akira, S. Issei, T. Tsuyosi, S. Yoshihiro and I. Un, Effect of whole body vibration exercise and muscle strengthening, balance, and walking exercise on walking ability in the elderly, The Keio Journal of Medicine $\mathbf{5 6}$ (2007), 28-33.

[16] S.R. Kang, K.S. Han, C.H. Yu and T. K. Kwon, Comparative analysis of basal physical fitness and muscle function in relation to muscle balance pattern using rowing machine, Bio-Medical Engineering Materials and Engineering 24 (2014), 2425-2435.

[17] C.H. Yu, C.U. Hong, S.R. Kang and T. K. Kwon, Analysis of basal physical fitness and lumbar muscle function according to indoor horse riding exercise, Bio-Medical Engineering Materials and Engineering 24 (2014), 2395-2405.

[18] M. Roelants, S. Verschueren, C. Delecluse, O. Levin and V. Stijnen, Whole body vibration induced increase in leg muscle activity during different squat exercises, Journal of Strength and Conditioning Research 20 (2006), 124-129.

[19] R. Anderson, C. Countney and E. Carmeli, EMG analysis of the vastus medialis/vastus lateralis muscles utilizing the unloaded narrow and wide stance squats, Journal of Sports Rehabilitation 7 (1998), 236-247.

[20] M. Roelants, M.P. Sabine, S. Verschueren, C. Delecluse, O. Levin and V. Stijnen, Whole-body-vibration-induce increase in leg muscle activity during different squat exercises, Journal of Strength and Conditioning Research 20 (2006), 124-129.

[21] K.S. Han, S.H. Shin, C.H. Yu and T. K. Kwon, Postural responses during the various frequencies of anteroposterior perturbation, Bio-Medical Engineering Materials and Engineering 24 (2014), 2537-2545.

[22] S. Torvinen, P. Kannus, H. Sievanen, T.A.H. Jarvinen, M. Pasanen, S. Kontulainen, T.L.N. Jarvinen, M. Jaravinen, P. Oja and I. Vuori, Effect of a vibration exposure on muscular performance and body balance, Randomized cross-over study, Clinical Physiology Functional Imaging 22 (2002), 145-152.

[23] J.A. Stewart, D.J. Cochrane and R.H. Morton, Differential effect of whole body vibration duration on knee extensor strength, Journal of Science and Medicine in Sport 12 (2009), 50-53.

[24] C.H. Yu, S.R. Kang and T. K. Kwon, Fundamental study of lower limb muscle activity using an angled whole body vibration exercise instrument, Bio-Medical Engineering Materials and Engineering 24 (2014), 2437-2445.

[25] P.M. Siu, B.T. Tam, D.H. Chow, J.Y. Guo, T.P. Huang, Y.P. Zheng and S.H. Won, Immediate effect of 2 different whole body vibration frequencies on muscle peak torque and stiffness, Archives of Physical Medicine and Rehabilitation 91 (2010), 1608-1615.

[26] S. Maeda, N.J. Mansfield and N. Shibata, Evaluation of subjective responses to whole body vibration exposure: Effect of frequency content, International Journal of Industrial Ergonomics 38 (2008), 509-515.

[27] C.H. Yu, S.R. Kang, K. Kim, H.C. Jeong and T. K. Kwon, Effect of recovery from muscle strength imbalance in lower limb using four point weight bearing reduction system, Bio-Medical Engineering Materials and Engineering 24 (2014), $2475-2483$

[28] A. Remaud, C. Cornu and A. Guevel, Agonist Muscle activity and antagonist muscle co-activity levels during standardized isotonic and isokinetic knee extensions, Journal of Electromyography and Kinesiology 19 (2009), 449-458.

[29] E.S. Go, K.M. Kim, D.J. Lee and N.S. Joo, Whole body vibration effects on body composition in the postmenopausal Korean obese women: plot study, Korean Journal of Family Medicine 32 (2011), 399-405.

[30] S.S. Rees, A.J. Murphy and M.L. Watsford, Effects of Whole body vibration exercise on lower extremity muscle strength and power in an older population: A randomized clinical trial, Journal of American Physical Therapy Association 88 (2012), 462-470. 